\title{
HIGH-RESOLUTION SPECTROSCOPY OF EXTREMELY METAL-POOR STARS IN THE LEAST EVOLVED GALAXIES: LEO IV*
}

\author{
Joshua D. Simon ${ }^{1}$, Anna Frebel ${ }^{2}$, Andrew McWilliam ${ }^{1}$, Evan N. Kirby ${ }^{3,4}$, And Ian B. Thompson ${ }^{1}$ \\ ${ }^{1}$ Observatories of the Carnegie Institution of Washington, 813 Santa Barbara Street, Pasadena, CA 91101, USA; jsimon@ociw.edu, andy@ociw.edu, ian@ociw.edu \\ ${ }^{2}$ Harvard-Smithsonian Center for Astrophysics, Cambridge, MA 02138, USA; afrebel@ cfa.harvard.edu \\ ${ }^{3}$ Department of Astronomy, California Institute of Technology, 1200 E. California Blvd., MS 249-17, Pasadena, CA 91125, USA; enk@astro.caltech.edu \\ Received 2010 January 18; accepted 2010 April 16; published 2010 May 19
}

\begin{abstract}
We present high-resolution Magellan/MIKE spectroscopy of the brightest star in the ultra-faint dwarf galaxy Leo IV. We measure an iron abundance of $[\mathrm{Fe} / \mathrm{H}]=-3.2$, adding to the rapidly growing sample of extremely metal-poor (EMP) stars being identified in Milky Way satellite galaxies. The star is enhanced in the $\alpha$ elements $\mathrm{Mg}, \mathrm{Ca}$, and $\mathrm{Ti}$ by $\sim 0.3$ dex, very similar to the typical Milky Way halo abundance pattern. All of the light and iron-peak elements follow the trends established by EMP halo stars, but the neutron-capture elements Ba and Sr are significantly underabundant. These results are quite similar to those found for stars in the ultra-faint dwarfs Ursa Major II, Coma Berenices, Boötes I, and Hercules, suggesting that the chemical evolution of the lowest-luminosity galaxies may be universal. The abundance pattern we observe is consistent with predictions for nucleosynthesis from a Population III supernova explosion. The extremely low metallicity of this star also supports the idea that a significant fraction $(\gtrsim 10 \%)$ of the stars in the faintest dwarfs have metallicities below $[\mathrm{Fe} / \mathrm{H}]=-3.0$.
\end{abstract}

Key words: galaxies: dwarf - galaxies: individual (Leo IV) - Local Group - stars: abundances

Online-only material: color figure

\section{INTRODUCTION}

The chemical abundance patterns of the most metal-poor stars provide a unique fossil record of star formation, chemical evolution, and supernova (SN) nucleosynthesis in the early universe. Until recently, such studies were limited to the stellar halo of the Milky Way because nearby dwarf galaxies appeared to lack sufficiently metal-poor stars (Helmi et al. 2006). Just over a year ago, the first extremely metal-poor (EMP) stars with $[\mathrm{Fe} / \mathrm{H}] \leqslant-3.0$ were discovered in several of the Milky Way's lowest-luminosity companions (Kirby et al. 2008). Since then, the number of known EMP stars in nearby dwarf galaxies has been expanding rapidly (Frebel et al. 2010b, 2010a; Cohen \& Huang 2009; Aoki et al. 2009; Norris et al. 2010).

Because the ultra-faint dwarfs host such incredibly tiny stellar populations $\left(L \lesssim 10^{4} L_{\odot}\right)$, they represent particularly attractive targets for chemical abundance studies. These galaxies should have hosted only a few $\mathrm{SNe}$, and the individual chemical signatures of those explosions may be revealed in their oldest stars (e.g., Koch et al. 2008). Moreover, they were likely some of the first objects to collapse in the early universe (Bovill \& Ricotti 2009), and the lack of star formation at later times means that evidence of the nucleosynthetic processes operating at high redshift should be preserved.

In a previous paper, we presented high-resolution spectra of six stars in the ultra-faint dwarfs Ursa Major II (UMa II) and Coma Berenices (ComBer), showing that both galaxies have very low metallicities, substantial iron abundance spreads, and overall abundance patterns similar to that of the Milky Way halo (Frebel et al. 2010b). Here we report spectroscopy of the brightest star (and the only one accessible to current telescopes at high spectral resolution) in the slightly more luminous galaxy Leo IV. In Section 2, we describe Leo IV and our observations.

\footnotetext{
* This paper includes data gathered with the $6.5 \mathrm{~m}$ Magellan Telescopes located at Las Campanas Observatory, Chile.

4 Hubble Fellow.
}

We present our abundance analysis in Section 3 and then discuss the implications of our results for the chemical evolution of the faintest galaxies in Section 4. In Section 5, we briefly summarize our findings and conclude.

\section{OBSERVATIONS AND DATA REDUCTION}

\subsection{Properties of Leo IV}

Leo IV was discovered as an overdensity of resolved stars in the fifth data release of the Sloan Digital Sky Survey (SDSS; Adelman-McCarthy et al. 2007) by Belokurov et al. (2007). Medium-resolution spectroscopy by Simon \& Geha (2007, hereafter SG07) demonstrated that Leo IV has stellar kinematics and metallicities that are characteristic of dwarf galaxies, but as the most poorly studied object in the SG07 sample its overall properties were not well constrained. Follow-up analysis of the SG07 spectra by Kirby et al. (2008) revealed that Leo IV has the lowest mean metallicity of any galaxy known, at $[\mathrm{Fe} / \mathrm{H}]=-2.58 \pm 0.08$, with a very large internal metallicity spread of $0.75 \mathrm{dex}$. Subsequently, photometric studies by Martin et al. (2008), Sand et al. (2009), Moretti et al. (2009), and de Jong et al. (2010) refined the size (128 $\pm 26 \mathrm{pc})$, absolute magnitude $\left(M_{V}=-5.7 \pm 0.3\right)$, and distance $(154 \pm 5 \mathrm{kpc})$ of the galaxy. SG07 identified a single bright red giant star, SDSSJ113255.99-003027.8 (hereafter referred to as Leo IVS1), in Leo IV at $V=19.2$, with the next brightest confirmed member nearly a magnitude fainter.

\subsection{Observations}

We observed Leo IV-S1 on 2009 February 18-20 with the Magellan Inamori Kyocera Echelle (MIKE) spectrograph (Bernstein et al. 2003) on the Clay Telescope. The observations were made with a $1^{\prime \prime}$ slit, producing a spectral resolution of $R=28,000$ on the blue side $(\lambda<5000 \AA)$ and a resolution of $R=22,000$ on the red side $(\lambda>5000 \AA)$. The CCD was binned $3 \times 3$ to reduce read noise for such a faint target, yielding a final 


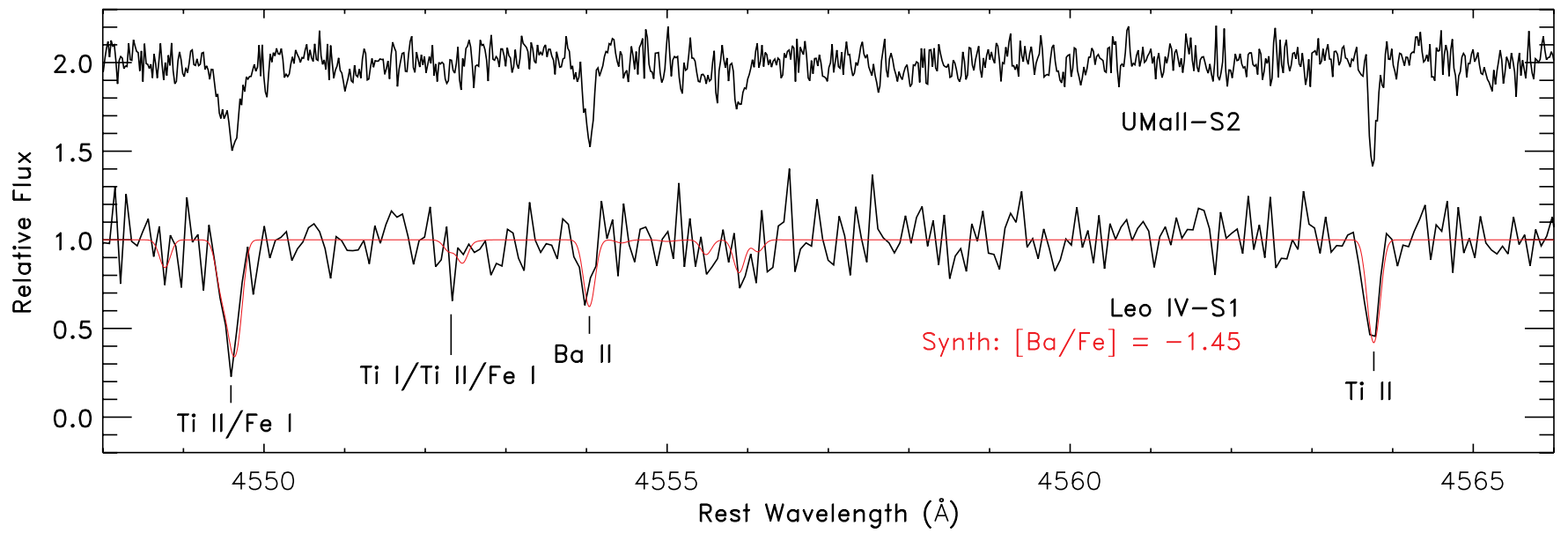

Figure 1. Spectrum of Leo IV-S1 around the Ba II $\lambda 4554 \AA$ Aine. Despite the low S/N at these wavelengths, Ba is clearly detected. Lines of Ti and Fe are also marked. The red line is a synthetic spectrum from MOOG using the derived atmospheric parameters and abundance ratios, and the spectrum of UMaII-S2 $\left(T_{\text {eff }}=4600 \mathrm{~K}\right.$, $[\mathrm{Fe} / \mathrm{H}]=-3.23)$ from Frebel et al. (2010b) is shown above for comparison.

(A color version of this figure is available in the online journal.)

dispersion of $\sim 0.07 \AA \mathrm{pixel}^{-1}$ in the blue and $\sim 0.12 \AA \mathrm{pixel}^{-1}$ in the red (i.e., sampling slightly better than the Nyquist rate). A temporary, lower-efficiency detector was used because of the failure of the MIKE blue CCD in 2008 November. The total integration time was $8.67 \mathrm{hr}$ (individual exposures were either 40 or 55 minutes) under mostly excellent observing conditions.

\subsection{Data Reduction}

The data were reduced using the latest version of the MIKE pipeline introduced by Kelson (2003). Frames from each night were reduced together, and then the spectra from the individual nights were co-added at the end. The final spectrum was normalized in IRAF, ${ }^{5}$ and each order was analyzed separately. Because of the target star's faintness, the signal-to-noise ratio $(\mathrm{S} / \mathrm{N})$ achieved is modest: $\mathrm{S} / \mathrm{N}=10 \mathrm{pixel}^{-1}$ at $4500 \AA$, $\mathrm{S} / \mathrm{N}=25 \mathrm{pixel}^{-1}$ at $5500 \AA$, and $\mathrm{S} / \mathrm{N}=45 \mathrm{pixel}^{-1}$ at $6500 \AA$, comparable to that obtained for similarly faint stars by Koch et al. $(2008,2009)$. We measure a velocity of $130.9 \pm 1.1 \mathrm{~km} \mathrm{~s}^{-1}$, consistent with the previous measurement of $132.7 \pm 2.2 \mathrm{~km} \mathrm{~s}^{-1}$ from SG07, which suggests that Leo IV-S1 does not have a binary companion in a close enough orbit to affect its evolution or abundances.

\section{ABUNDANCE ANALYSIS}

\subsection{Line Measurements and Atmospheric Parameters}

Using a line list taken from McWilliam et al. (1995b) and Frebel et al. (2010b), we measured equivalent widths (EWs) with the IRAF task SPLOT. We detected $\sim 50 \mathrm{Fe}$ I lines, and between 1 and 10 lines for the following species: Fe II, Mg I, Ca I, Sc II, Ti I, Ti iI, Na I, Cri, Ni I, Sr II, and Ba II. A portion of the spectrum illustrating the detection of $\mathrm{Ba}$ is displayed in Figure 1, and the EWs of all measured lines are listed in Table 1.

Based on a combined photometric and spectroscopic analysis, Kirby et al. (2008) estimated $T_{\text {eff }}=4330 \mathrm{~K}, \log g=1.0$, $\xi=1.6 \mathrm{~km} \mathrm{~s}^{-1}$, and $[\mathrm{Fe} / \mathrm{H}]=-2.9$ for Leo IV-S1. Starting

\footnotetext{
5 IRAF is distributed by the National Optical Astronomy Observatory, which is operated by the Association of Universities for Research in Astronomy under cooperative agreement with the National Science Foundation.
}

with these parameters, we constructed a one-dimensional planeparallel Kurucz model atmosphere (Kurucz 1992) and then iteratively redetermined the stellar parameters using the $\mathrm{Fe}$ I lines with the 2009 version of MOOG (Sneden 1973). We first established the microturbulent velocity by minimizing the trend of $\mathrm{Fe}$ I abundance with EW. The derived value was $\xi=3.2 \mathrm{~km} \mathrm{~s}^{-1}$, which would be quite high for the less luminous stars that are frequently observed in the Milky Way halo and globular clusters, but is comparable to values obtained for cool EMP giants from low $\mathrm{S} / \mathrm{N}$ spectra ${ }^{6}$ in a number of other studies (McWilliam et al. 1995b; Koch et al. 2008; Aoki et al. 2009; Frebel et al. 2010a).

Next, we determined the effective temperature. Leo IV-S1 is unfortunately too faint to have been detected by the Two Micron All Sky Survey (2MASS), so the reddest available color is $V-I$ (converted from the SDSS magnitudes using the Jordi et al. 2006 transformations). The color-temperature relation of Alonso et al. (1999) predicts $T_{\text {eff }}=4330 \mathrm{~K}$ using either $B-V$ (from Moretti et al. 2009) or $V-I$. At this temperature, there is still a weak negative trend of $\mathrm{Fe}$ I abundance with excitation potential, as noted for similar stars by Norris et al. (2010, and references therein), perhaps indicating deviations from local thermodynamic equilibrium. Forcing Fe I excitation balance and deriving $T_{\text {eff }}$ from the spectrum alone would lead to a lower value $(\sim 4200 \mathrm{~K})$.

Ideally, the surface gravity would be set by imposing ionization balance on the $\mathrm{Fe}$ I and $\mathrm{Fe}$ II lines. Unfortunately, with the relatively low $\mathrm{S} / \mathrm{N}$ and resolution of our spectra, very few Fe II lines were detectable, and they are all either weak features and/or in low $\mathrm{S} / \mathrm{N}$ regions of the spectrum. We therefore do not consider any of our Fe II measurements (which span nearly an order of magnitude in abundance) to be very reliable. We instead resorted to the more basic technique of applying the Stefan-Boltzmann law and Newton's law of gravitation to calculate the gravity. The apparent $r$ magnitude of Leo IV-S1 after correcting for interstellar reddening of $E(B-V)=0.025 \mathrm{mag}$ (Schlegel et al. 1998) is $r=18.76$. Given a distance modulus for Leo IV of 20.94 mag (Moretti et al. 2009), the absolute magnitude is $M_{r}=-2.18$. Using isochrones from

\footnotetext{
6 Even if the $\mathrm{S} / \mathrm{N}$ results in $\xi$ being overestimated, the effect on the abundances is small $(\sim 0.1 \mathrm{dex})$.
} 
Table 1

Equivalent Width Measurements

\begin{tabular}{|c|c|c|c|c|c|}
\hline Element & $\begin{array}{c}\lambda \\
(\AA)\end{array}$ & $\begin{array}{c}\chi \\
(\mathrm{eV})\end{array}$ & $\log g f$ & $\begin{array}{c}\text { EW } \\
(\mathrm{mA})\end{array}$ & $\log \epsilon$ \\
\hline $\mathrm{NaI}$ & 5889.973 & 0.00 & 0.100 & 220.0 & 3.28 \\
\hline $\mathrm{NaI}$ & 5895.940 & 0.00 & -0.200 & 160.0 & 2.84 \\
\hline Mg I & 4571.102 & 0.00 & -5.670 & 64.0 & 4.47 \\
\hline $\mathrm{Mg}_{\mathrm{I}}$ & 4703.003 & 4.34 & -0.520 & 75.0 & 5.11 \\
\hline $\mathrm{Mg}_{\mathrm{I}}$ & 5172.698 & 2.71 & -0.380 & 231.0 & 4.66 \\
\hline $\mathrm{Mg}_{\mathrm{I}}$ & 5183.619 & 2.72 & -0.160 & 256.0 & 4.66 \\
\hline $\mathrm{Mg}_{\mathrm{I}}$ & 5528.418 & 4.34 & -0.341 & 74.0 & 4.76 \\
\hline $\mathrm{Ca} \mathrm{I}$ & 5588.764 & 2.52 & 0.358 & 47.0 & 3.39 \\
\hline $\mathrm{Ca} \mathrm{I}$ & 5594.471 & 2.52 & 0.097 & 22.0 & 3.22 \\
\hline $\mathrm{Ca} I$ & 6102.727 & 1.88 & -0.770 & 38.0 & 3.51 \\
\hline $\mathrm{Ca} \mathrm{I}$ & 6122.226 & 1.89 & -0.320 & 62.0 & 3.38 \\
\hline $\mathrm{Ca} I$ & 6162.180 & 1.90 & -0.090 & 90.0 & 3.47 \\
\hline $\mathrm{Ca} I$ & 6439.083 & 2.52 & 0.390 & 61.0 & 3.45 \\
\hline Sc II & 5031.024 & 1.36 & -0.400 & 41.0 & 0.27 \\
\hline Sc II & 5526.821 & 1.77 & 0.020 & 42.0 & 0.33 \\
\hline Sc II & 5657.880 & 1.51 & -0.600 & 18.0 & 0.14 \\
\hline Ti I & 5039.964 & 0.02 & -1.130 & 25.0 & 1.76 \\
\hline Ti I & 5210.392 & 0.05 & -0.884 & 58.0 & 2.01 \\
\hline Ti II & 4417.723 & 1.16 & -1.430 & 82.0 & 2.13 \\
\hline Ti II & 4443.812 & 1.08 & -0.700 & 127.0 & 1.95 \\
\hline Ti II & 4468.500 & 1.13 & -0.600 & 120.0 & 1.79 \\
\hline Ti II & 4501.278 & 1.12 & -0.750 & 136.0 & 2.15 \\
\hline Ti II & 4563.766 & 1.22 & -0.960 & 122.0 & 2.24 \\
\hline Ti II & 4571.982 & 1.57 & -0.530 & 111.0 & 2.12 \\
\hline Ti II & 4589.953 & 1.24 & -1.790 & 70.0 & 2.37 \\
\hline Ti II & 5185.908 & 1.89 & -1.350 & 26.0 & 2.02 \\
\hline Ti II & 5336.794 & 1.58 & -1.700 & 63.0 & 2.48 \\
\hline Ti II & 5381.028 & 1.57 & -2.080 & 21.0 & 2.19 \\
\hline Cr I & 4254.346 & 0.00 & -0.114 & 141.0 & 1.92 \\
\hline $\mathrm{Cr} \mathrm{I}$ & 5345.807 & 1.00 & -0.980 & 26.0 & 2.08 \\
\hline $\mathrm{Fe} I$ & 4447.728 & 2.22 & -1.339 & 77.0 & 4.30 \\
\hline Fe I & 4494.573 & 2.20 & -1.136 & 74.0 & 4.01 \\
\hline Fe I & 4871.325 & 2.86 & -0.362 & 60.0 & 3.81 \\
\hline $\mathrm{Fe} I$ & 4872.144 & 2.88 & -0.567 & 91.0 & 4.44 \\
\hline $\mathrm{Fe} I$ & 4890.763 & 2.87 & -0.394 & 71.0 & 3.99 \\
\hline Fe I & 4891.502 & 2.85 & -0.111 & 83.0 & 3.83 \\
\hline $\mathrm{Fe} I$ & 4994.138 & 0.91 & -2.956 & 71.0 & 3.93 \\
\hline $\mathrm{Fe} I$ & 5041.076 & 0.96 & -3.086 & 86.0 & 4.29 \\
\hline Fe I & 5041.763 & 1.48 & -2.203 & 114.0 & 4.46 \\
\hline Fe I & 5049.827 & 2.28 & -1.355 & 67.0 & 4.09 \\
\hline Fe I & 5123.730 & 1.01 & -3.058 & 115.0 & 4.66 \\
\hline $\mathrm{Fe} I$ & 5127.368 & 0.91 & -3.249 & 81.0 & 4.31 \\
\hline $\mathrm{Fe} I$ & 5133.699 & 4.18 & 0.140 & 23.0 & 4.38 \\
\hline $\mathrm{Fe} I$ & 5150.852 & 0.99 & -3.037 & 113.0 & 4.58 \\
\hline $\mathrm{Fe} I$ & 5151.917 & 1.01 & -3.321 & 70.0 & 4.38 \\
\hline $\mathrm{Fe}_{\mathrm{I}}$ & 5166.284 & 0.00 & -4.123 & 94.0 & 4.08 \\
\hline Fe I & 5171.610 & 1.48 & -1.721 & 118.0 & 3.98 \\
\hline $\mathrm{Fe}_{\mathrm{I}}$ & 5191.465 & 3.04 & -0.551 & 47.0 & 4.01 \\
\hline $\mathrm{Fe} I$ & 5192.353 & 3.00 & -0.421 & 83.0 & 4.27 \\
\hline Fe I & 5194.949 & 1.56 & -2.021 & 120.0 & 4.41 \\
\hline $\mathrm{Fe}_{\mathrm{I}}$ & 5216.283 & 1.61 & -2.082 & 91.0 & 4.17 \\
\hline Fe I & 5225.534 & 0.11 & -4.755 & 82.0 & 4.72 \\
\hline Fe I & 5232.952 & 2.94 & -0.057 & 109.0 & 4.13 \\
\hline Fe I & 5250.216 & 0.12 & -4.938 & 68.0 & 4.76 \\
\hline Fe I & 5281.798 & 3.04 & -0.833 & 48.0 & 4.29 \\
\hline $\mathrm{Fe} I$ & 5283.629 & 3.24 & -0.524 & 40.0 & 4.13 \\
\hline $\mathrm{Fe} I$ & 5302.307 & 3.28 & -0.720 & 55.0 & 4.57 \\
\hline $\mathrm{Fe}_{\mathrm{I}}$ & 5307.369 & 1.61 & -2.912 & 48.0 & 4.48 \\
\hline Fe I & 5324.191 & 3.21 & -0.103 & 99.0 & 4.39 \\
\hline Fe I & 5497.526 & 1.01 & -2.825 & 128.0 & 4.49 \\
\hline $\mathrm{Fe}_{\mathrm{I}}$ & 5501.477 & 0.96 & -3.046 & 110.0 & 4.41 \\
\hline $\mathrm{Fe} I$ & 5506.791 & 0.99 & -2.789 & 142.0 & 4.60 \\
\hline $\mathrm{Fe} I$ & 5569.631 & 3.42 & -0.500 & 54.0 & 4.49 \\
\hline $\mathrm{Fe}_{\mathrm{I}}$ & 5572.851 & 3.40 & -0.275 & 57.0 & 4.27 \\
\hline $\mathrm{Fe}_{\mathrm{I}}$ & 5615.658 & 3.33 & 0.050 & 82.0 & 4.14 \\
\hline
\end{tabular}

Table 1

(Continued)

\begin{tabular}{|c|c|c|c|c|c|}
\hline Element & $\begin{array}{c}\lambda \\
(\AA)\end{array}$ & $\begin{array}{c}\chi \\
(\mathrm{eV})\end{array}$ & $\log g f$ & $\begin{array}{c}\text { EW } \\
(\mathrm{mA})\end{array}$ & $\log \epsilon$ \\
\hline $\mathrm{Fe}_{\mathrm{I}}$ & 6136.624 & 2.45 & -1.410 & 82.0 & 4.37 \\
\hline $\mathrm{Fe}_{\mathrm{I}}$ & 6137.702 & 2.59 & -1.346 & 66.0 & 4.32 \\
\hline $\mathrm{Fe}_{\mathrm{I}}$ & 6191.571 & 2.43 & -1.416 & 95.0 & 4.48 \\
\hline $\mathrm{Fe} I$ & 6219.287 & 2.20 & -2.448 & 40.0 & 4.58 \\
\hline $\mathrm{Fe}_{\mathrm{I}}$ & 6230.736 & 2.56 & -1.276 & 60.0 & 4.13 \\
\hline $\mathrm{Fe}_{\mathrm{I}}$ & 6252.565 & 2.40 & -1.767 & 59.0 & 4.39 \\
\hline $\mathrm{Fe}_{\mathrm{I}}$ & 6265.141 & 2.18 & -2.550 & 21.0 & 4.31 \\
\hline $\mathrm{Fe}_{\mathrm{I}}$ & 6335.337 & 2.20 & -2.180 & 43.0 & 4.34 \\
\hline $\mathrm{Fe} I$ & 6393.612 & 2.43 & -1.576 & 68.0 & 4.33 \\
\hline $\mathrm{Fe}_{\mathrm{I}}$ & 6400.009 & 3.60 & -0.290 & 40.0 & 4.24 \\
\hline $\mathrm{Fe}_{\mathrm{I}}$ & 6411.658 & 3.65 & -0.595 & 25.0 & 4.35 \\
\hline Fe I & 6421.360 & 2.28 & -2.014 & 44.0 & 4.28 \\
\hline $\mathrm{Fe} I$ & 6430.856 & 2.18 & -1.946 & 75.0 & 4.44 \\
\hline $\mathrm{Fe}_{\mathrm{I}}$ & 6494.994 & 2.40 & -1.239 & 100.0 & 4.28 \\
\hline $\mathrm{Fe} I$ & 6677.997 & 2.69 & -1.420 & 58.0 & 4.38 \\
\hline $\mathrm{Fe} I$ & 6750.164 & 2.42 & -2.621 & 13.0 & 4.43 \\
\hline Fe II & 4923.930 & 2.89 & -1.260 & 84.0 & 4.02 \\
\hline Fe II & 5018.450 & 2.89 & -1.110 & 120.0 & 4.35 \\
\hline Fe II & 5197.560 & 3.23 & -2.220 & 44.0 & 4.83 \\
\hline Fe II & 5276.000 & 3.20 & -2.010 & 67.0 & 4.88 \\
\hline Fe II & 6456.391 & 3.90 & -2.075 & 21.0 & 5.00 \\
\hline $\mathrm{Ni}$ I & 6643.638 & 1.68 & -2.300 & 48.0 & 3.56 \\
\hline $\mathrm{Ni}$ I & 6767.784 & 1.83 & -2.170 & 20.0 & 3.15 \\
\hline Sr II & 4215.539 & 0.00 & -0.170 & 120.0 & -1.34 \\
\hline Ba II & 4554.036 & 0.00 & 0.160 & 62.0 & -2.46 \\
\hline
\end{tabular}

Table 2

Stellar Parameters for Leo IV-S

\begin{tabular}{lcc}
\hline \hline Parameter & $\begin{array}{c}\text { Fiducial } \\
\text { Model }\end{array}$ & $\begin{array}{c}\text { Spectroscopic } \\
\text { Model }\end{array}$ \\
\hline$M_{r}$ & -2.18 & -2.18 \\
$T_{\text {eff }}(\mathrm{K})$ & 4330 & 4200 \\
$\log g$ & 1.0 & 0.0 \\
$\xi\left(\mathrm{km} \mathrm{s}^{-1}\right)$ & 3.2 & 3.2 \\
\hline
\end{tabular}

Girardi et al. (2004), we estimate a bolometric correction of -0.11 mag for stars of similar evolutionary state, yielding a luminosity of $637 L_{\odot}$. For a mass of $0.8 M_{\odot}$, the corresponding surface gravity is $\log g=1.0$, varying only weakly with the assumed temperature $\left(\Delta \log g=-0.05\right.$ dex for $\left.\Delta T_{\text {eff }}=100 \mathrm{~K}\right)$. This value for the gravity produces an Fe II abundance that is $\sim 0.3$ dex higher than the Fe I abundance, but again, we do not regard the Fe II measurement as reliable. A much lower gravity of $\log g \sim 0$ would be required to bring $[\mathrm{Fe} \mathrm{I} / \mathrm{H}]$ and $[\mathrm{Fe}$ II $/ \mathrm{H}]$ into better agreement. Our final atmospheric parameters are therefore $T_{\text {eff }}=4330 \mathrm{~K}, \log g=1.0$, and $\xi=3.2 \mathrm{~km} \mathrm{~s}^{-1}$, but we also derive abundances for the purely spectroscopic values of $T_{\text {eff }}=4200 \mathrm{~K}, \log g=0.0$, and $\xi=3.2 \mathrm{~km} \mathrm{~s}^{-1}$ for comparison (see Table 2).

\subsection{Derived Abundances and Uncertainties}

We list the measured abundances from MOOG in Table 3. Because of the low quality of our Fe II measurement, we adopt $[\mathrm{Fe} / \mathrm{H}]=[\mathrm{Fe} \mathrm{I} / \mathrm{H}]$ to calculate $[\mathrm{X} / \mathrm{Fe}]$ values for ionized species as well as neutral ones. Note that we use the new Asplund et al. (2009) solar abundances (with $\log \epsilon(\mathrm{Fe})=7.50$ ).

Since the photometric and spectroscopic solutions for $T_{\text {eff }}$ and $\log g$ are not entirely consistent, assessing the impact that our 
Table 3

Chemical Abundances of Leo IV-S1

\begin{tabular}{|c|c|c|c|c|c|c|}
\hline \multirow[t]{2}{*}{ Species } & \multicolumn{5}{|c|}{ Fiducial Model } & \multirow{2}{*}{$\frac{\text { Spectroscopic Model }}{\text { Abundance Ratio }}$} \\
\hline & Abundance Ratio & $\log \epsilon(X)$ & $\mathrm{N}_{\text {lines }}$ & $\sigma_{\text {stat }}{ }^{\mathrm{a}}$ & $\sigma_{\text {sys }}{ }^{\mathrm{b}}$ & \\
\hline$[\mathrm{Fe} \mathrm{I} / \mathrm{H}]$ & -3.19 & 4.31 & 50 & 0.03 & 0.27 & -3.09 \\
\hline$[\mathrm{Fe} \mathrm{II} / \mathrm{H}]$ & $-2.88:$ & 4.62: & 5 & 0.19 & 0.14 & $-2.89:$ \\
\hline$[\mathrm{C} / \mathrm{Fe}]$ & $<-0.08$ & $<5.16$ & Synth & $\ldots$ & $\ldots$ & $<0.32$ \\
\hline$[\mathrm{Na} \mathrm{I} / \mathrm{Fe}]$ & 0.01 & 3.06 & 2 & 0.28 & 0.09 & -0.04 \\
\hline$[\mathrm{Mg} \mathrm{I} / \mathrm{Fe}]$ & 0.32 & 4.73 & 5 & 0.12 & 0.09 & 0.62 \\
\hline$[\mathrm{Ca}$ I/Fe] & 0.25 & 3.40 & 6 & 0.05 & 0.09 & 0.26 \\
\hline [Ti I/Fe] & 0.13 & 1.89 & 2 & 0.16 & 0.07 & 0.08 \\
\hline [Ti II/Fe] & 0.38 & 2.14 & 10 & 0.06 & 0.28 & 0.40 \\
\hline$[\mathrm{Sc}$ II $/ \mathrm{Fe}]$ & 0.29 & 0.25 & 3 & 0.06 & 0.33 & 0.00 \\
\hline [Cr I/Fe] & -0.45 & 2.00 & 2 & 0.10 & 0.11 & -0.44 \\
\hline [Ni I/Fe] & 0.32 & 3.35 & 2 & 0.26 & 0.07 & 0.16 \\
\hline$[\mathrm{Sr} \mathrm{II} / \mathrm{Fe}]$ & -1.02 & -1.34 & 1 & $\ldots$ & 0.26 & -0.96 \\
\hline$[\mathrm{Ba}$ II $/ \mathrm{Fe}]$ & -1.45 & -2.46 & 1 & $\ldots$ & 0.24 & -1.56 \\
\hline
\end{tabular}

Notes. All abundance ratios $[\mathrm{X} / \mathrm{Fe}]$ (including ionized species) are calculated relative to Fe I.

a Statistical uncertainties are defined as the standard error of the mean of abundances of individual lines (accounting for small sample sizes where fewer than 10 lines are used).

b Systematic uncertainties refer to quadrature sums of the changes listed in Table 4 relative to Fe I for each species.

Table 4

Abundance Uncertainties

\begin{tabular}{|c|c|c|c|c|}
\hline Species & $\begin{array}{c}\Delta \log \epsilon(X) \\
\text { for } T_{\text {eff }}+150 \mathrm{~K}\end{array}$ & $\begin{array}{c}\Delta \log \epsilon(X) \\
\text { for } \log g-0.5 \mathrm{dex}\end{array}$ & $\begin{array}{c}\Delta \log \epsilon(X) \\
\text { for }[\mathrm{M} / \mathrm{H}]+0.3 \mathrm{dex}\end{array}$ & $\begin{array}{c}\Delta \log \epsilon(X) \\
\text { for } \xi+0.3 \mathrm{~km} \mathrm{~s}^{-1}\end{array}$ \\
\hline $\mathrm{Fe}$ I & 0.23 & 0.13 & -0.03 & -0.04 \\
\hline Fe II & -0.08 & -0.10 & 0.01 & -0.05 \\
\hline $\mathrm{NaI}$ & 0.24 & 0.14 & -0.07 & -0.12 \\
\hline $\mathrm{Mg}_{\mathrm{I}}$ & 0.19 & 0.21 & -0.05 & -0.06 \\
\hline $\mathrm{Ca} \mathrm{I}$ & 0.15 & 0.10 & -0.02 & -0.01 \\
\hline Ti I & 0.29 & 0.14 & -0.04 & -0.01 \\
\hline Ti II & -0.01 & 0.00 & 0.01 & -0.06 \\
\hline Sc II & 0.02 & -0.12 & 0.02 & -0.01 \\
\hline CrI & 0.25 & 0.21 & -0.09 & -0.07 \\
\hline $\mathrm{Ni}$ I & 0.25 & 0.07 & -0.01 & -0.01 \\
\hline Sr II & -0.01 & 0.07 & -0.04 & -0.11 \\
\hline Ba II & 0.07 & -0.04 & 0.02 & -0.02 \\
\hline
\end{tabular}

choices for these parameters have on the derived abundances is important. An estimate of the systematic uncertainties can be obtained from the abundance differences between the two sets of stellar parameters. To quantify these further, we also vary the atmospheric parameters one at a time by approximately their uncertainties and examine the resulting abundance changes. The parameter uncertainties are set by considering how large a change is allowed by the Fe I abundances for $\xi$ and $T_{\text {eff }}$, and assigning reasonable uncertainty levels for the gravity and overall metallicity: $\Delta T_{\text {eff }}=+150 \mathrm{~K}, \Delta \log g=-0.5 \mathrm{dex}$, $\Delta[\mathrm{M} / \mathrm{H}]=+0.3 \mathrm{dex}$, and $\Delta \xi=+0.3 \mathrm{~km} \mathrm{~s}^{-1}$. The systematic uncertainties we derive are listed in Tables 3 and 4. Over this range of parameters, the maximum iron abundance we obtain is $[\mathrm{Fe} / \mathrm{H}]=-2.96$, so we can confidently conclude that Leo IV-S1 is indeed an EMP star.

\section{DISCUSSION}

\subsection{The Frequency of EMP Stars in Dwarf Galaxies}

Including Leo IV-S1, there are now detailed abundance studies (with individually determined atmospheric parameters) for 10 stars in the ultra-faint dwarfs (Koch et al. 2008; Frebel et al. 2010b; Norris et al. 2010). The highest metallicity star included in these studies has $[\mathrm{Fe} / \mathrm{H}]=-2.0$ (Koch et al. 2008), and four have metallicities below $[\mathrm{Fe} / \mathrm{H}]=-3.0$. As noted by Frebel et al. (2010b), with the exception of Boo-1137 from Norris et al. (2010), these stars have been selected independent of their metallicities: the sole selection criterion (by necessity) is their apparent magnitude. The 33\% success rate (three out of nine, after excluding Boo-1137) at finding EMP stars strongly suggests that a substantial fraction of the stars in these systems have extremely low metallicities (Kirby et al. 2008; Salvadori \& Ferrara 2009). In order to obtain three EMP stars in a random drawing out of a sample of nine, the EMP fraction must be at least $10 \%$ at the $95 \%$ confidence level. The results of Norris et al. (2008) that 4 of 16 stars observed at medium resolution in Boo I (including Boo-1137) have metallicities below $[\mathrm{Fe} / \mathrm{H}]=-3.0$ provide further support for this case. As the observed data sets increase further, it therefore seems likely that many more EMP stars, and perhaps stars with even lower metallicities, will be identified. Provided that one is willing to invest the telescope time to obtain high-resolution spectra of 18th-19th magnitude stars, the ultrafaint dwarfs may be the most promising targets for increasing samples of stars with $[\mathrm{Fe} / \mathrm{H}]<-3.5$ and studying the fossil clues left behind by the first generation of stars. 

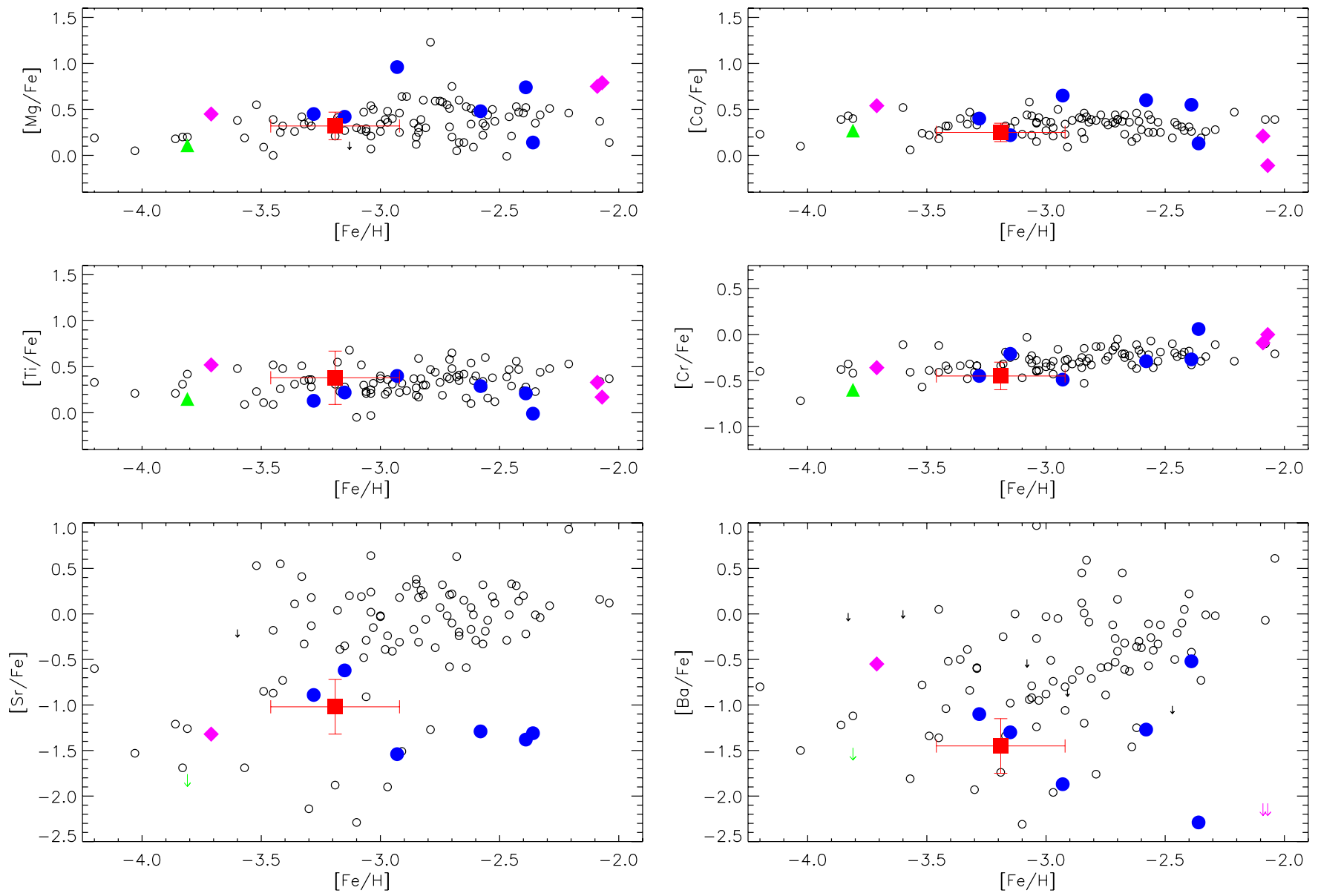

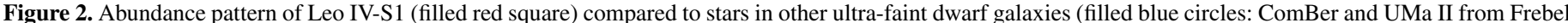

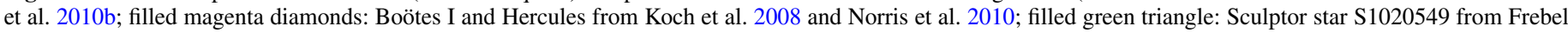

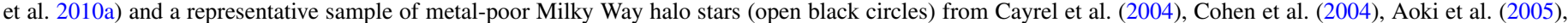

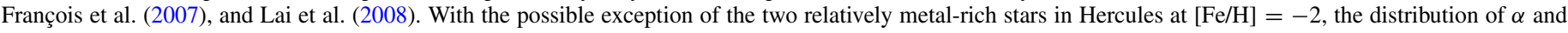

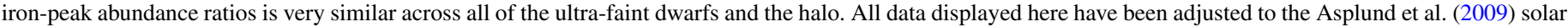
abundance scale.

\subsection{Abundance Patterns in the Ultra-faint Dwarfs}

The abundances of light and iron-peak elements in Leo IV-S1 match closely those that we measured in UMa II and ComBer. The $\alpha$ elements $\mathrm{Mg}, \mathrm{Ca}$, and $\mathrm{Ti}$ are each enhanced by $\sim 0.3 \mathrm{dex}$ compared to the solar ratios, identical within the uncertainties to those of the two EMP stars in UMa II. Sc, $\mathrm{Cr}$, and $\mathrm{Ni}$ in Leo IV-S1 also agree with the measured abundances of UMa II below $[\mathrm{Fe} / \mathrm{H}]=-3$. Only a conservative $[\mathrm{C} / \mathrm{Fe}]$ limit could be obtained for Leo IV-S1, indicating that the star is not strongly $\mathrm{C}$-enriched. These similarities suggest that whatever process is responsible for producing elements from $\mathrm{Na}$ at least through the iron peak in the ultra-faint dwarfs seems to be nearly universal, yielding similar abundances in almost every star examined so far. The only exception is the ratio of hydrostatic to explosive $\alpha$ elements (e.g., $[\mathrm{Mg} / \mathrm{Ca}]$ ), which is strongly enhanced in a fraction of the ultra-faint dwarf stars (Koch et al. 2008; Frebel et al. 2010b; Feltzing et al. 2009). As found by Frebel et al. (2010b) and illustrated in Figure 2, this common abundance pattern in ultra-faint dwarf stars (including Leo IV-S1) also agrees well with that of EMP stars in the Milky Way halo (e.g., Cayrel et al. 2004; Lai et al. 2008).

Moreover, Leo IV-S1 continues the trend of unusually low neutron-capture abundances in the ultra-faint dwarfs (Koch et al. 2008; Frebel et al. 2010b), with $[\mathrm{Ba} / \mathrm{Fe}]=-1.45$ and $[\mathrm{Sr} / \mathrm{Fe}]=-1.02$. Unlike the lighter species, for heavy elements the ultra-faints as a whole do not agree with typical halo behavior; the halo has a higher mean abundance and spans a larger range of [nc/Fe] at similar metallicities (Figure 2; also see François et al. 2007; Lai et al. 2008). Stars in the brighter dwarf spheroidals (dSphs) generally have roughly solar abundances of $\mathrm{Ba}$ and $\mathrm{Eu}$, although a few of the most metal-poor stars in those galaxies show a similar deficiency of heavy elements as the ultra-faint dwarfs (Fulbright et al. 2004; Frebel et al. 2010a). This distinction from both the halo and the classical dSphs suggests that the heavy elements may be produced differently in the ultra-faint dwarfs than in their brighter counterparts (at least at later times).

Abundance measurements of the few strongly $r$-processenhanced EMP stars in the halo indicate that SNe that produce $r$-process elements in large quantities must be rare (e.g., McWilliam et al. 1995a) or inefficient at dispersing those elements into the surrounding gas. It has been suggested that core-collapse $\mathrm{SNe}$ over a narrow mass range are the astrophysical site for the main $r$-process, perhaps in the lowest-mass $\mathrm{SNe}$ (8-10 $M_{\odot}$; e.g., Qian \& Wasserburg 2003; Wanajo et al. 2003). If the enrichment of all of the ultra-faint dwarfs is a result of randomly sampling $\mathrm{SNe}$ from a common initial mass function 
(IMF), and assuming the main $r$-process to be the dominant source for the observed neutron-capture elements, then most dwarfs that incompletely sample the $\mathrm{SN}$ mass function will show deficient $[\mathrm{Sr} / \mathrm{Fe}]$ and $[\mathrm{Ba} / \mathrm{Fe}]$ ratios because $r$-process $\mathrm{SNe}$ are rare. A small fraction, however, should contain relatively $r$-process-rich EMP stars. Since the IMF of the first stars is expected to be top heavy (e.g., Yoshida et al. 2006), the low $r$-process abundances we observe could arise naturally if $r$-process elements are predominantly made by these lowermass $\mathrm{SNe}$.

Despite the overall broad similarities with the other ultrafaints, possible signs of stochasticity in the abundance patterns of the faintest dwarfs are also evident in the data that have been acquired over the past few years. Two stars in Hercules and one each in Boo I (Feltzing et al. 2009) and Draco (Fulbright et al. 2004) exhibit very high $[\mathrm{Mg} / \mathrm{Ca}]$ ratios that are argued to result from small numbers of $\mathrm{SNe}$ and the resulting incomplete sampling of the IMF (Koch et al. 2008). The Hercules stars also have extremely low (nearly unprecedented; see Figure 2) upper limits for $[\mathrm{Ba} / \mathrm{Fe}]$, while $\mathrm{Ba}$ has been detected in every star observed so far in UMa II, ComBer, and Leo IV, despite much lower overall metallicities. It may be noteworthy that it is the most luminous ultra-faint dwarfs that seem to contain these unusual signatures, but larger samples in all of these galaxies are needed before drawing strong conclusions.

\subsection{Supernovae and Nucleosynthesis in Leo IV}

Heger \& Woosley (2008) have shown that Population III $\mathrm{SNe}$ from initially metal-free massive stars can produce an elemental abundance pattern similar to that measured by Cayrel et al. (2004) for EMP halo stars. In a similar study, Tominaga et al. (2007) concluded that Pop III hypernovae with unusually high explosion energies are necessary to match the Cayrel et al. (2004) data. The agreement between the abundances of Leo IV-S1 and the Cayrel et al. sample suggests that some form of Pop III nucleosynthesis may be able to explain the chemical abundances of Leo IV as well. In Figure 3, we demonstrate the quality of the match that can be obtained between the observed abundances and the models; the best fit found by comparisons with the grid of models from Heger \& Woosley (2008) is for a low-mass $\left(\sim 10 M_{\odot}\right) \mathrm{SN}$ with an average explosion energy. Higher-mass hypernova explosions can also provide acceptable fits.

Because the number of stars in Leo IV is so small $\left(L_{V}=\right.$ $\left.14000 L_{\odot}\right)$, the metal content of the entire galaxy is extremely low. For example, given the mean metallicity determined by Kirby et al. (2008) and assuming a stellar mass-to-light ratio of $1 M_{\odot} / L_{\odot}$, Leo IV contains just $0.042 M_{\odot}$ of Fe. Since this is comparable to the amount of Fe produced by the best-fitting Pop III SN models (Heger \& Woosley 2008), if Leo IV was indeed enriched by such explosions then a single $S N$ may be enough to have produced all of the observed heavy elements. It is also possible, of course, that multiple SNe contributed to the chemical evolution of the galaxy if most of the metals were blown out via winds rather than having been incorporated into subsequent generations of stars. Nevertheless, we tentatively conclude that Leo IV-S1 may reflect the nucleosynthetic yields of the first Pop III star that the galaxy formed, at a time when its gas content was $\sim 4 \times 10^{4} M_{\odot}$. Other ultra-faint dwarfs therefore might reveal the abundance patterns of $\mathrm{SNe}$ with different masses, consistent with recent observations of Hercules (Koch et al. 2008).

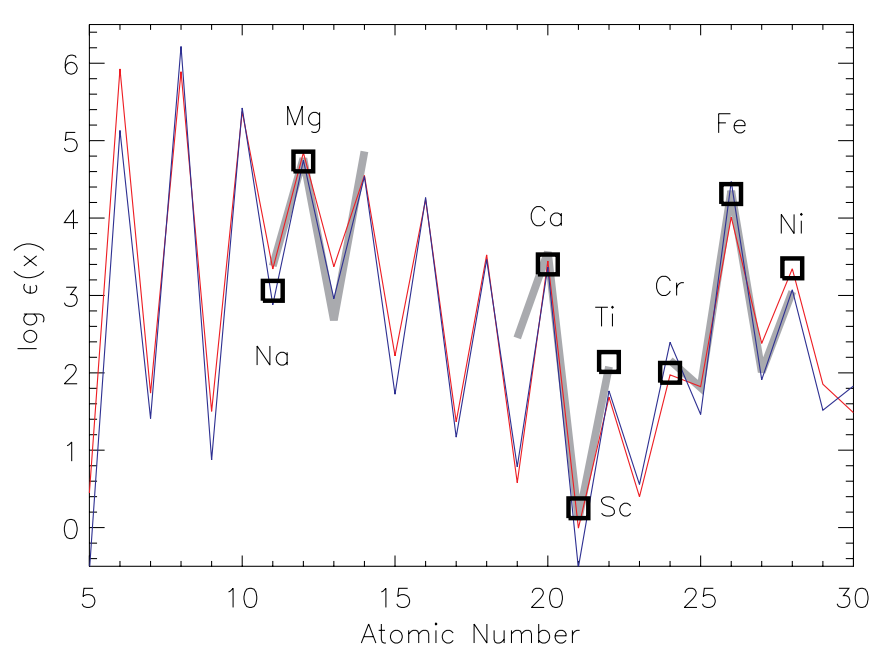

Figure 3. Observed abundance pattern of Leo IV-S1 (black squares) compared to the Cayrel et al. (2004) halo sample (thick gray line) at similar metallicities and Population III SN nucleosynthesis models from Heger \& Woosley (2008). A Heger \& Woosley "standard" $10.2 M_{\odot}$ model (red line), diluted by $4700 M_{\odot}$ of primordial gas, reproduces the overall abundance pattern fairly well. A high explosion energy $29.5 M_{\odot}$ Heger \& Woosley SN (added to $2.6 \times 10^{5} M_{\odot}$ of hydrogen) predicts a similar pattern for many elements but with discrepant abundances for $\mathrm{Sc}$ and $\mathrm{Cr}$ (blue line)

\section{SUMMARY AND CONCLUSIONS}

We have carried out a high-resolution abundance analysis for the brightest star in the ultra-faint dwarf galaxy Leo IV. With $[\mathrm{Fe} / \mathrm{H}]=-3.2$, Leo IV-S1 adds to the rapidly increasing sample of EMP stars in dwarf galaxies. The low metallicity of Leo IV-S1 provides further support for the hypothesis that a substantial fraction $(\gtrsim 10 \%)$ of the stars in the faintest dwarfs lie in the EMP regime.

The abundance pattern in Leo IV is extremely similar to that found in both the other ultra-faint dwarfs and the metalpoor Milky Way halo. As suggested by Frebel et al. (2010b), this excellent agreement demonstrates that the metal-poor end of the halo metallicity distribution could have been formed in galaxies like the ultra-faint dwarfs. The only exception to the close match between the halo and the ultra-faint dwarfs is the neutron-capture elements, which still appear somewhat lower in the dwarfs, but more measurements of these elements are needed.

Interestingly, the most metal-poor stars in some of the brighter dSphs seem to share the same chemical signature of $\alpha$-enhancement and neutron-capture depletion (Fulbright et al. 2004; Frebel et al. 2010a), although the abundances in those systems deviate substantially at higher metallicities (Shetrone et al. 2003; Venn et al. 2004). The similarity between the abundances of Leo IV-S1 and other dwarfs such as UMa II, ComBer, Boo I, and Sculptor suggests that the initial enrichment in many galaxies may have been universal. Differences between the abundances in the faintest dwarfs (ComBer, UMa II, and Leo IV) and the stars in somewhat more luminous systems, on the other hand, could point to stochastic chemical variations. Finally, we show that the abundance pattern of Leo IV-S1 is consistent with Population III SN models, raising the possibility that Leo IV was enriched by some of the first stars.

We thank Dan Kelson for assistance with the data reduction, Steve Shectman for observing suggestions, Alexander Heger for extensive help with comparisons to theoretical SN models, and the referee for a helpful report. J.D.S gratefully acknowledges 
the support of a Vera Rubin Fellowship provided by the Carnegie Institution of Washington, and A.F. that of a Clay Fellowship administered by the Smithsonian Astrophysical Observatory. This work was also supported by NASA through Hubble Fellowship grant HST-HF-01233.01 awarded to E.N.K. by the Space Telescope Science Institute, which is operated by the Association of Universities for Research in Astronomy, Inc., for NASA, under contract NAS 5-26555. This research made use of NASA's Astrophysics Data System Bibliographic Services.

Facilities: Magellan:Clay (MIKE)

\section{REFERENCES}

Adelman-McCarthy, J. K., et al. 2007, ApJS, 172, 634

Alonso, A., Arribas, S., \& Martínez-Roger, C. 1999, A\&AS, 140, 261

Aoki, W., et al. 2005, ApJ, 632, 611

Aoki, W., et al. 2009, A\&A, 502, 569

Asplund, M., Grevesse, N., Sauval, A. J., \& Scott, P. 2009, ARA\&A, 47, 481

Belokurov, V., et al. 2007, ApJ, 654, 897

Bernstein, R., Shectman, S. A., Gunnels, S. M., Mochnacki, S., \& Athey, A. E. 2003, Proc. SPIE, 4841, 1694

Bovill, M. S., \& Ricotti, M. 2009, ApJ, 693, 1859

Cayrel, R., et al. 2004, A\&A, 416, 1117

Cohen, J. G., \& Huang, W. 2009, ApJ, 701, 1053

Cohen, J. G., et al. 2004, ApJ, 612, 1107

de Jong, J. T. A., Martin, N. F., Rix, H.-W., Smith, K. W., Jin, S., \& Macció, A. V. 2010, ApJ, 710, 1664

Feltzing, S., Eriksson, K., Kleyna, J., \& Wilkinson, M. I. 2009, A\&A, 508, L1

François, P., et al. 2007, A\&A, 476, 935

Frebel, A., Kirby, E. N., \& Simon, J. D. 2010a, Nature, 464, 72

Frebel, A., Simon, J. D., Geha, M., \& Willman, B. 2010b, ApJ, 708, 560

Fulbright, J. P., Rich, R. M., \& Castro, S. 2004, ApJ, 612, 447

Girardi, L., Grebel, E. K., Odenkirchen, M., \& Chiosi, C. 2004, A\&A, 422, 205
Heger, A., \& Woosley, S. E. 2008, ApJ, submitted (arXiv:0803.3161)

Helmi, A., et al. 2006, ApJ, 651, L121

Jordi, K., Grebel, E. K., \& Ammon, K. 2006, A\&A, 460, 339

Kelson, D. D. 2003, PASP, 115, 688

Kirby, E. N., Simon, J. D., Geha, M., Guhathakurta, P., \& Frebel, A. 2008, ApJ, 685, L43

Koch, A., Côté, P., \& McWilliam, A. 2009, A\&A, 506, 729

Koch, A., McWilliam, A., Grebel, E. K., Zucker, D. B., \& Belokurov, V. 2008, ApJ, 688, L13

Kurucz, R. L. 1992, in IAU Symp. 149, The Stellar Populations of Galaxies, ed B. Barbuy \& A. Renzini (Dordrecht: Kluwer), 225

Lai, D. K., Bolte, M., Johnson, J. A., Lucatello, S., Heger, A., \& Woosley, S. E. 2008, ApJ, 681, 1524

Martin, N. F., de Jong, J. T. A., \& Rix, H.-W. 2008, ApJ, 684, 1075

McWilliam, A., Preston, G. W., Sneden, C., \& Searle, L. 1995a, AJ, 109 2757

McWilliam, A., Preston, G. W., Sneden, C., \& Shectman, S. 1995b, AJ, 109 2736

Moretti, M. I., et al. 2009, ApJ, 699, L125

Norris, J. E., Gilmore, G., Wyse, R. F. G., Wilkinson, M. I., Belokurov, V., Evans, N. W., \& Zucker, D. B. 2008, ApJ, 689, L113

Norris, J. E., Yong, D., Gilmore, G., \& Wyse, R. F. G. 2010, ApJ, 711, 350

Qian, Y.-Z., \& Wasserburg, G. J. 2003, ApJ, 588, 1099

Salvadori, S., \& Ferrara, A. 2009, MNRAS, 395, L6

Sand, D. J., Seth, A., Olszewski, E. W., Willman, B., Zaritsky, D., \& Kallivayalil, N. 2009, ApJ, submitted (arXiv:0911.5352)

Schlegel, D. J., Finkbeiner, D. P., \& Davis, M. 1998, ApJ, 500, 525

Shetrone, M., Venn, K. A., Tolstoy, E., Primas, F., Hill, V., \& Kaufer, A. 2003, AJ, 125,684

Simon, J. D., \& Geha, M. 2007, ApJ, 670, 313 (SG07)

Sneden, C. 1973, ApJ, 184, 839

Tominaga, N., Umeda, H., \& Nomoto, K. 2007, ApJ, 660, 516

Venn, K. A., Irwin, M., Shetrone, M. D., Tout, C. A., Hill, V., \& Tolstoy, E. 2004, AJ, 128, 1177

Wanajo, S., Tamamura, M., Itoh, N., Nomoto, K., Ishimaru, Y., Beers, T. C., \& Nozawa, S. 2003, ApJ, 593, 968

Yoshida, N., Omukai, K., Hernquist, L., \& Abel, T. 2006, ApJ, 652, 6 